\title{
PRECISION MASSES OF THE LOW-MASS BINARY SYSTEM GJ 623
}

\author{
Frantz Martinache, ${ }^{1}$ James P. Lloyd, ${ }^{1}$ Michael J. Ireland, ${ }^{2}$ Ryan S. Yamada, ${ }^{1}$ and Peter G. Tuthill ${ }^{3}$ \\ Received 2006 December 4; accepted 2007 February 7
}

\begin{abstract}
We have used aperture masking interferometry and adaptive optics (AO) at the Palomar 200 inch telescope to obtain precise mass measurements of the binary M dwarf GJ 623. AO observations spread over 3 yr combined with a decade of radial velocity measurements constrain all orbital parameters of the GJ 623 binary system accurately enough to critically challenge the models. The dynamical masses measured are $m_{1}=0.371 \pm 0.015 M_{\odot}(4 \%)$ and $m_{2}=$ $0.115 \pm 0.0023 M_{\odot}(2 \%)$ for the primary and the secondary, respectively. Models are not consistent with color and mass, requiring very low metallicities.
\end{abstract}

Subject headings: binaries: general — stars: luminosity function, mass function — techniques: interferometric

Online material: color figures

\section{INTRODUCTION}

The mass of a star, along with its metallicity and age, is the fundamental parameter that determines its position along an evolutionary track. Even if binarity, rotation, magnetic fields, and other parameters also affect stellar interiors, what is known as the Vogt-Russel theorem remains an important rule, and the mass an essential parameter of stellar evolution. Binary systems offer the ideal test to infer dynamical masses independent of the use of a stellar model, such as those of Baraffe et al. (1998), or an empirical mass-luminosity $(M-L)$ relation. Such relations, available for both visible (Henry et al. 1999) and near-infrared (Delfosse et al. 2000), are important astrophysical tools, fairly well constrained for intermediate-mass stars. However, the solar neighborhood is dominated by low-mass stars, in both number and total mass (Henry 1998), and as dust condenses in the atmospheres of these cool stars, the models meet new unknowns. There are also puzzles in the stellar structure of $\mathrm{M}$ dwarfs and the extrapolation of these models to substellar objects remains untested. Below $0.6 M_{\odot}$, both $M-L$ relations and models will benefit from modelindependent determinations of high-precision stellar masses, which can be achieved by combining radial velocimetry measurements and high angular resolution imaging (Ségransan et al. 2000; Delfosse et al. 2004). The complementarity of the two techniques yields substantial benefits, even in the regime where the time baseline of the observations is shorter than the orbital period (Eisner \& Kulkarni 2002).

We have observed known M dwarf binaries with precise radial velocity measurements published by Nidever et al. (2002). These late-type $\mathrm{M}$ dwarfs are ideal observing targets for imaging with the PALAO adaptive optics (AO) system and the PHARO infrared camera (Hayward et al. 2001), optimized for the near-infrared bands. At small angular separation (i.e., $<2 \lambda / d$ ), the sensitivity of the detection of faint companions can be improved by combining AO with aperture-masking interferometry (Pravdo et al. 2006; Lloyd et al. 2006). This paper provides the astrometry of the binary system GJ 623, successfully observed with both AO and $\mathrm{AO}+$ aperture masking. Combined with the radial velocity mea-

\footnotetext{
1 Department of Astronomy, Cornell University, Ithaca, NY.

2 Division of Geological and Planetary Sciences, California Institute of Technology, Pasadena, CA.

3 School of Physics, University of Sydney, Sydney, NSW, Australia.
}

surement, the astrometry provides precise dynamical masses (better than $2 \%$ ) of the GJ 623 binary system. Combined with the $J$, $H, K$ photometry, this measurement adds new constraints to the models and $M-L$ relations.

\section{THE OBSERVATIONS}

\subsection{The Primary}

GJ 623 (aka LHS 417, HIP 80346) is a high proper motion M2.5 dwarf 8 pc from the Sun. It is a long-known astrometric binary, first characterized by Lippincott \& Borgman (1978). GJ 623 has proven to be an ideal test for different observational techniques, such as radial velocimetry (Marcy \& Moore 1989; Nidever et al. 2002) and speckle interferometry (McCarthy \& Henry 1987). This binary system has also been directly imaged in the visible with the COSTAR-corrected HST FOC (Faint Object Camera; Barbieri et al. 1996).

Our observations of GJ 623 were performed with the PHARO instrument on the Palomar 200 inch $(5 \mathrm{~m})$ telescope and with the NIRC2 instrument on the Keck II telescope. The companion of GJ 623 was detected in six observing runs: 2003 September, 2004 June, 2005 January, and 2006 February, at Palomar, using the $J, H$ and $K_{s}$ filters, and 2006 June and August at Keck, with the $1.58 \mu \mathrm{m} H_{\text {cont }}$ filter. Tables 1 and 2, respectively, gather the astrometric and photometric measurements made at Palomar and Keck.

Four of our observations consist of conventional direct imaging with AO. These images were dark subtracted, flat-fielded, and analyzed with a custom IDL program, using the latest PHARO and NIRC2 plate scale and orientation characterization by Metchev \& Hillenbrand (2004). The location of the companion (angular separation and position angle) and the contrast ratio are precisely determined with a cross-correlation of the images. Not surprisingly, doubling the diameter of the telescope (cf. the Keck data points) significantly improves the precision of the measurements and roughly reduces the error bars by a factor 3 .

The data also include observations in aperture-masking interferometry with AO. This technique (Tuthill et al. 2000), recently described by Lloyd et al. (2006), is appropriate to the detection of faint companion at small angular separation (typically less than $2 \lambda / D)$, where direct $\mathrm{AO}$ imaging has so far proven difficult.

Indeed, present AO systems focus on achieving high contrast at moderate angular separation $>4 \lambda / D$. Below this limit, the variance of the speckle background dominates the photon noise by 
TABLE 1

Astrometric Measurements at Palomar and Keck: Angular Separation and Position Angle of GJ 623B

\begin{tabular}{|c|c|c|c|c|}
\hline $\begin{array}{c}\text { Julian Date } \\
(-2,450,000)\end{array}$ & Band & $\begin{array}{c}\text { Pupil } \\
\text { (see text) }\end{array}$ & $\begin{array}{c}\text { Separation } \\
\text { (mas) }\end{array}$ & $\begin{array}{l}\text { P.A. } \\
\text { (deg) }\end{array}$ \\
\hline $2896.6 .$. & $K_{s}$ & 9 hole & $240.4 \pm 9.7$ & $79.31 \pm 2.0$ \\
\hline $3163.8 \ldots \ldots$ & {$\left[\mathrm{Fe}_{\mathrm{II}}\right]$} & 18 hole & $340.5 \pm 20.4$ & $49.16 \pm 4.3$ \\
\hline $3402.1 .$. & $K_{s}$ & Full aperture & $350.7 \pm 2.2$ & $28.66 \pm 0.5$ \\
\hline 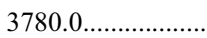 & $J, H, K_{s}$ & Full aperture & $257.1 \pm 3.1$ & $-12.76 \pm 0.6$ \\
\hline $3909.5 \ldots$. & $H_{\text {cont }}$ & Nirc2 & $176.7 \pm 1.1$ & $318.2 \pm 0.2$ \\
\hline 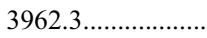 & $H_{\text {cont }}$ & Nirc2 & $138.7 \pm 0.9$ & $295.4 \pm 0.2$ \\
\hline
\end{tabular}

several orders of magnitude (Racine et al. 1999). This prevents us from calibrating the point-spread function (PSF) with a precision sufficient to discriminate faint companions from the bright star's residual speckles.

This issue can be evaded by using interferometric techniques. By sampling a few spatial frequencies only, a mask located in the pupil plane permits us to completely decompose the PSF into a finite set of Fourier components. Non-redundancy of the baselines passed by the mask ensures that each frequency is sampled only once and the visibilities can be used to form closure phases (Baldwin et al. 1986; Readhead et al. 1988; Nakajima et al. 1989). This observable rejects both atmospheric noise and calibration errors of the wave front sensor. The only drawback is the transmission of such a mask: between $5 \%$ and $15 \%$ for the ones used at Palomar. However, in the speckle noise limited regime, light loss does not result in a loss of sensitivity.

Our analysis also uses a HST observation dating to 1994, and originally published by Barbieri et al. (1996): an angular distance of $330 \pm 20$ mas and a position angle of $7.0^{\circ} \pm 2.6^{\circ}$ on 1994 June 11. Even though this is inferior to the precision achieved with Palomar and Keck, which are much larger telescopes, the fact that this observation was performed more than $10 \mathrm{yr}$ ago adds an important constraint on the period of the binary.

From the 2MASS catalog, the apparent magnitudes of GJ $623 \mathrm{AB}$ are $J=6.638 \pm 0.024, H=6.141 \pm 0.021$, and $K=$ $5.915 \pm 0.023$.

The Hipparcos parallax $\pi=124.34 \pm 1.16$ mas originally published by Perryman et al. (1997) was based on the radial velocimetry measurement of Marcy \& Moore (1989). The parallax was recalculated by Jancart et al. (2005) after the publication of an improved radial velocity curve by Nidever et al. (2002). It is this revised parallax $\pi=125.81 \pm 1.19$ mas (D. Pourbaix 2006 , private communication) that we adopt here. The following absolute magnitudes can be deduced for GJ 623AB: $M_{J}=7.137 \pm$ $0.052, M_{H}=6.640 \pm 0.051$, and $M_{K}=6.414 \pm 0.052$.

TABLE 2

Photometric Measurements of GJ 623B

\begin{tabular}{|c|c|c|}
\hline $\begin{array}{c}\text { Julian Date } \\
(-2,450,000)\end{array}$ & Filter & $\Delta$ mag \\
\hline $2896.6 \ldots \ldots \ldots \ldots \ldots$ & $K_{s}$ & $3.051 \pm 0.826$ \\
\hline 3163.8.............................. & $\mathrm{Fe}$ II & $2.903 \pm 0.476$ \\
\hline 3402.1........................ & $K_{s}$ & $2.604 \pm 0.047$ \\
\hline \multirow[t]{3}{*}{ 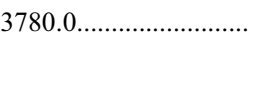 } & $J$ & $2.691 \pm 0.038$ \\
\hline & $H$ & $2.860 \pm 0.039$ \\
\hline & $K_{s}$ & $2.789 \pm 0.014$ \\
\hline $3909.5 \ldots \ldots \ldots \ldots \ldots$ & $H_{\text {cont }}$ & $2.794 \pm 0.033$ \\
\hline $3962.3 \ldots \ldots \ldots \ldots \ldots \ldots$ & $H_{\text {cont }}$ & $2.781 \pm 0.016$ \\
\hline
\end{tabular}

TABLE 3

Orbital Elements

\begin{tabular}{|c|c|c|}
\hline Parameter & Nidever & This Work \\
\hline 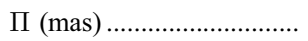 & $\ldots$ & $125.81 \pm 1.19$ \\
\hline$a(\mathrm{AU})$ & $\ldots$ & $1.894 \pm 0.019$ \\
\hline$\alpha$ (mas) & $\ldots$ & $237.28 \pm 0.88$ \\
\hline 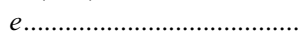 & $0.67 \pm 0.01$ & $0.631 \pm 0.002$ \\
\hline$i(\operatorname{deg}) \ldots \ldots \ldots \ldots$ & $\ldots$ & $154.0 \pm 0.1$ \\
\hline$\Omega_{1}(\mathrm{deg}) \ldots \ldots \ldots \ldots \ldots \ldots$ & $\ldots$ & $98.5 \pm 0.47$ \\
\hline$\omega_{0}(\mathrm{deg})$ & $251 \pm 1$ & $248.68 \pm 0.46$ \\
\hline 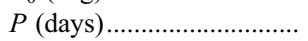 & $1366.1 \pm 0.4$ & $1365.6 \pm 0.3$ \\
\hline$T_{P}($ reduced JD) $\ldots \ldots \ldots \ldots \ldots$ & $1298 \pm 10$ & $1313.3 \pm 0.6$ \\
\hline$V_{0}\left(\mathrm{~km} \mathrm{~s}^{-1}\right) \ldots \ldots \ldots \ldots \ldots \ldots \ldots$ & $-27.654 \pm 0.3$ & $-27.729 \pm 0.005$ \\
\hline$A_{1}\left(\mathrm{kms}^{-1}\right) \ldots \ldots \ldots \ldots \ldots \ldots$ & $\ldots$ & $3.57 \pm 0.01$ \\
\hline
\end{tabular}

\section{METHOD: EXTRACTING ORBITAL PARAMETERS}

The starting point in the determination of the orbital parameters of the GJ 623 system is the set of radial velocity measurements published by Nidever et al. (2002) and available in the online version of the Ninth Catalogue of Spectroscopic Binary Orbits ${ }^{4}$ (Pourbaix et al. 2004). The parameters deduced from the Keplerian fit to the data are summarized in Table 3 . They have been directly used by Jancart et al. (2005) to reprocess the Hipparcos intermediate astrometric Data of van Leeuwen \& Evans (1998) for a complete characterization of the dynamical elements of the system.

The approach detailed in this paper consists of combining together, with no a priori assumptions, the original radial velocity data and our astrometric observations.

\subsection{Keplerian Orbits}

One uses the standard two-body solution to parameterize the location of the companion orbiting GJ 623A. To an observing date $t$, one associates an angle $M$ called the mean anomaly:

$$
M(t)=\frac{2 \pi}{P}\left(t-T_{P}\right),
$$

where $P$ and $T_{P}$, respectively, represent the orbital period and the epoch at the periastron passage. In the orbital plane, the $(x, y)$ coordinates can simply be expressed as a function of another angle, the eccentric anomaly $E$. It is the angle between the direction of the periastron and the current position of the companion, projected onto the ellipse's circumscribing circle perpendicularly to the major axis, measured at the center of the ellipse (cf. Fig. 1):

$$
\begin{aligned}
M(E) & =E-e \sin E \\
x(E) & =a(\cos E-e) \\
y(E) & =a \sqrt{1-e^{2}} \sin E,
\end{aligned}
$$

where $a$ and $e$, respectively, represent the semimajor axis and the eccentricity of the orbit. Equation (2) is called Kepler's equation. One solves this equation, i.e., finds the value of $E$ associated with a given $M$ by using the following classical iterative algorithm:

$$
\begin{aligned}
E_{0} & =M \\
E_{n+1} & =M+e \sin E_{n} .
\end{aligned}
$$

${ }^{4}$ See http://sb9.astro.ulb.ac.be. 


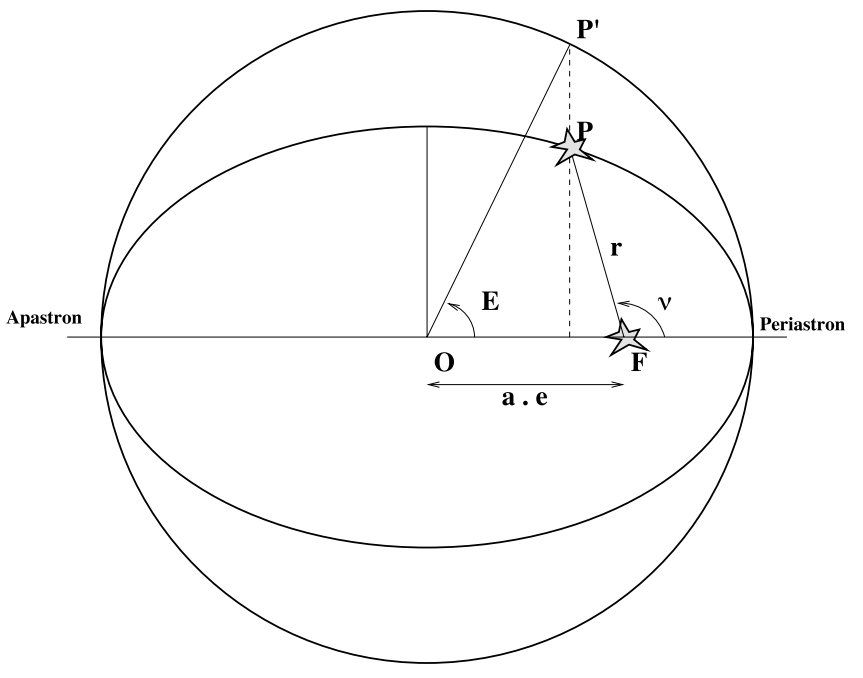

Fig. 1.-Parameterization of an elliptic orbit. Geometric relation between the eccentric anomaly $E$ and the true anomaly $\nu$. [See the electronic edition of the Journal for a color version of this figure.]

\subsubsection{Radial Velocity Orbital Models}

One can use the standard two-body solution (eqs. [3] and [4]) to compute the coordinates of the primary component in the orbital plane, relative to the center of mass of the system:

$$
\left[\begin{array}{l}
x_{P}(E) \\
y_{p}(E)
\end{array}\right]=\frac{m_{2}}{m_{T}}\left[\begin{array}{l}
x(E) \\
y(E)
\end{array}\right],
$$

where $m_{T}$ and $m_{2}$, respectively, stand for the total mass (primary+ secondary) and the mass of the secondary. In the orbital plane, the velocity vector can be calculated with the following partial derivative:

$$
V=\frac{\partial}{\partial t}\left(\begin{array}{l}
x \\
y
\end{array}\right)=\frac{\partial}{\partial E}\left(\begin{array}{l}
x \\
y
\end{array}\right) \frac{\partial E}{\partial M} \frac{\partial M}{\partial t} .
$$

This derivation leads to the following $x$ and $y$ components of the velocity:

$$
\left[\begin{array}{c}
v_{x}(E) \\
v_{y}(E)
\end{array}\right]=\frac{m_{2}}{m_{T}} \frac{2 \pi a}{P(1-e \cos E)}\left[\begin{array}{c}
-\sin E \\
\sqrt{1-e^{2}} \cos E
\end{array}\right] .
$$

The radial velocity (RV) is the component of this velocity projected on the line of sight. Its expression therefore involves both the argument of the periastron $\omega_{0}$ and the inclination of the system $i$. The $\mathrm{RV}$ is not sensitive to the orientation of the system on the sky, i.e., to the value of the argument of the ascending node $\Omega_{1}$.

$R V\left(E, \omega_{0}, i\right)=\left[v_{x}(E) \sin \omega_{0}+v_{y}(E) \cos \omega_{0}\right] \sin i+V_{0}$,

where $V_{0}$ is a constant term, the mean RV.

\subsubsection{Astrometric Orbital Models}

Unlike the case of RV data for which equations are expressed from the center of mass of the system, the astrometric measurements gathered in Table 1 provide the instant position of the companion relative to the primary. In this frame, the trajectory of the companion is an ellipse whose focus is the primary component. The parametric equation of this ellipse is

$$
r(\nu)=\frac{a\left(1-\epsilon^{2}\right)}{1+\epsilon \cos \nu},
$$

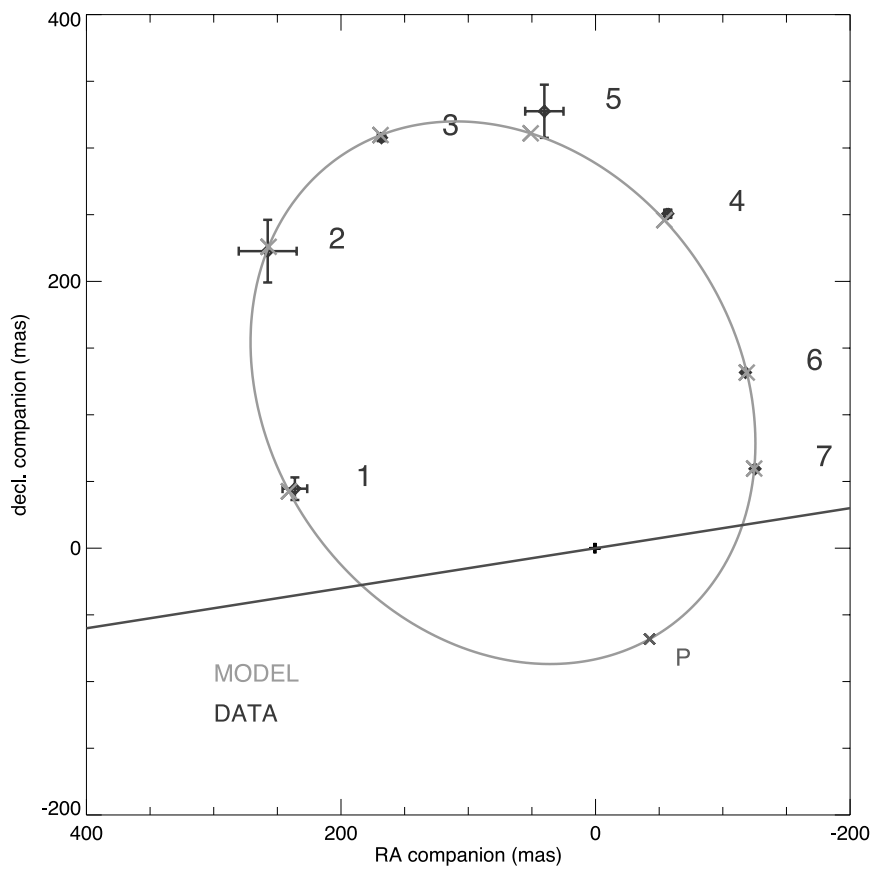

FIG. 2.-Orbit of GJ 623B. Measurements and associated uncertainties are represented by black points with error bars. Points 1-4: PHARO observations. Point 5: HST observations reported in Barbieri et al. (1996). Points 6-7: Nirc2 observations. Gray lines: Keplerian fit to the orbit. The straight line is the line of nodes, and the point $P$ marks the position of the periastron. [See the electronic edition of the Journal for a color version of this figure.]

where $a$ is the semimajor axis, $\epsilon$ the eccentricity $(0<\epsilon<1)$, and $\nu$ an angle called the true anomaly, which is the angle between the direction of the periastron and the current position of an object on its orbit ( $P$ in Fig. 1), measured at the focus of the ellipse.

Figure 1 illustrates the one-to-one correspondence between eccentric and true anomaly. The projection of the point $P$ on the major axis provides the following relation:

$$
r \cos \nu=a(\cos E-\epsilon) .
$$

Together, equations. (10) and (11) lead to the primary to secondary distance $r$ and the true anomaly $\nu$, as functions of the eccentric anomaly $E$ :

$$
\begin{aligned}
r & =a(1-e \cos E), \\
\cos \nu & =\frac{\cos E-\epsilon}{1-\epsilon \cos E} .
\end{aligned}
$$

At a given observing date $t$, one needs once more to solve Kepler's equation (cf. eq. [2]) to determine the corresponding eccentric anomaly $E$. The location of the companion along the orbit is provided by equations (12) and (13).

Contrary to the RV, which is the component of the velocity projected on the line of sight, one measures here the position of the companion projected on the celestial sphere. Right ascension, $\alpha$, and declination, $\delta$, of the secondary (relative to the primary) are given by the following relations:

$$
\begin{gathered}
\alpha=r\left[\cos \left(\nu+\omega_{0}\right) \sin \Omega_{1}+\sin \left(\nu+\omega_{0}\right) \cos i \cos \Omega_{1}\right], \\
\delta=r\left[\cos \left(\nu+\omega_{0}\right) \cos \Omega_{1}-\sin \left(\nu+\omega_{0}\right) \cos i \sin \Omega_{1}\right] . \\
\text { 3.2. } \chi^{2} \text { Fitting }
\end{gathered}
$$

We use the model presented in the previous section to fit a nine-parameter model to 26 observables. The observables are 

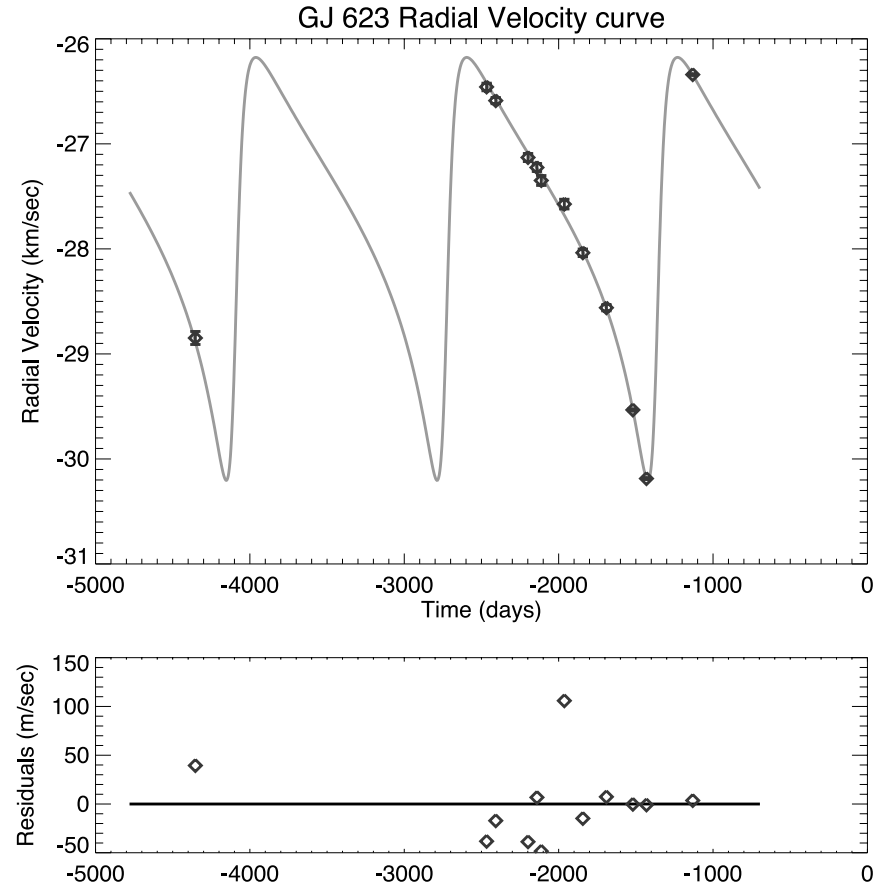

FIG. 3.-Radial velocity curve of GJ 623A. The measurements and associated uncertainties reported by Nidever et al. (2002) are represented by diamonds. The solid line represents the Keplerian orbit with the parameters derived by $\chi^{2}$ fitting. [See the electronic edition of the Journal for a color version of this figure.]

two coordinates for seven astrometric data points and 12 radial velocities. The nine parameters are the six orbital elements: the semimajor axis $a$, the eccentricity $e$, the longitude of the ascending node $\omega_{0}$, the inclination $i$, the argument of periastron $\Omega_{1}$, and the orbital period $P$, plus the RV offset $V_{0}$ and the semiamplitude of the RV curve $K_{1}$.

In the case of a conventional analysis of RV data, the main observable is the semiamplitude $K_{1}$, which once combined with the period and the eccentricity of the orbit, provides the mass function

$$
f\left(M_{1}, M_{2}, i\right)=\frac{M_{2}^{3} \sin i^{3}}{\left(M_{T}\right)^{2}} .
$$

This combined analysis RV+astrometry allows us to separate the geometrical effects from the semiamplitude. This produces another composite observable, somewhat simpler than $K_{1}$, a "pseudoamplitude," whose formal expression is

$$
A_{1}=\frac{M_{2}}{M_{T}} \frac{2 \pi a}{P} .
$$

This is the parameter that will be used to determine the dynamical masses of the two components of the binary.

The final result of this nine-parameter fit, with 17 degrees of freedom, is represented in Figure 2 for the astrometry and in Figure 3 for the radial velocimetry. Our solution exhibits a final reduced $\chi_{\nu}^{2}=1.03$, which is, despite the heterogeneity of the data, close enough to unity to ensure confidence in our estimation of the error bars. It is dominated by the velocimetry, despite having fewer measurements than the astrometry.

The confidence interval of each of the parameters is determined by analysis of the likelihood function. If we assume that the noise associated to our measurements is Gaussian, and that our parameters are independent, the likelihood can be approximated by

$$
L(\text { parameters }) \propto \exp \left(-\frac{\chi^{2}}{2}\right) .
$$

The computation of a nine-dimensional likelihood function requires a lot of CPU time. This difficulty may be circumvented by confining the search to a subset of the total space. This is achieved by fixing the values of certain parameters and calculating the joined likelihood for the remaining parameters. However, one needs to check a posteriori that the hypothesis of independent parameters is valid.

This analysis reveals that among the nine parameters, only the argument of the periastron $\omega_{0}$ and the longitude of the ascending node $\Omega_{1}$ exhibit significant correlation. These two parameters are constrained within $0.5^{\circ}$ (cf. Table 3). The hypothesis of independent parameters is valid, as expected from our good coverage in both radial velocimetry and astrometry. The uncertainty associated with each parameter is taken equal to the standard deviation of its associated likelihood function. The Gaussian-like likelihoods of the parameters used in the determination of the dynamical masses are shown in Figure 4.

\section{CHARACTERISTICS OF GJ 623AB}

The orbital parameters and the confidence intervals derived from the likelihood analysis are summarized in Table 3. This
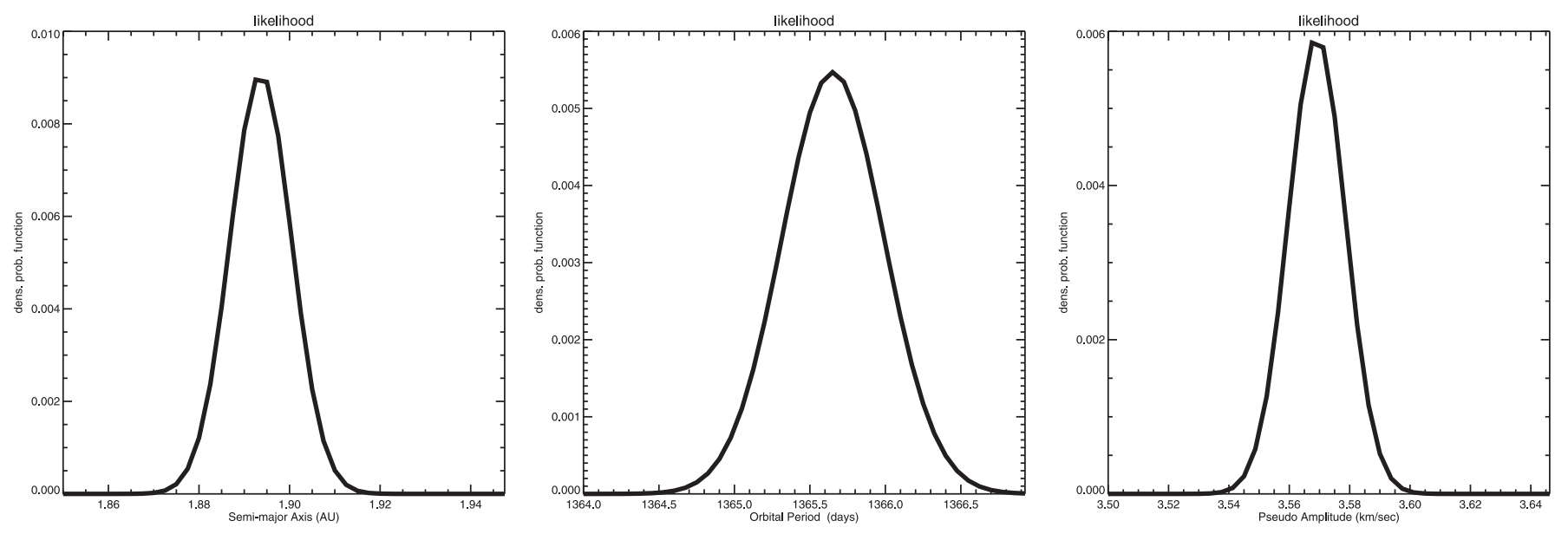

FIG. 4.-Likelihood functions of semimajor axis $a$, period $P$, and RV pseudoamplitude $A_{1}$, used to determine the dynamical masses of the GJ 623 system. 
TABLE 4

Dynamical Masses

\begin{tabular}{|c|c|}
\hline Quantity & $\begin{array}{l}\text { Value } \\
\left(M_{\odot}\right)\end{array}$ \\
\hline Total mass ................................ & $M_{T}=0.486 \pm 0.015$ \\
\hline Primary mass................................. & $M_{1}=0.371 \pm 0.015$ \\
\hline Secondary mass .......................... & $M_{2}=0.115 \pm 0.0023$ \\
\hline
\end{tabular}

combined analysis significantly improved the constraints on the epoch at periastron passage, from $T_{P}=2451298 \pm 10$ (Nidever et al. 2002) to the new value $T_{P}=2451313.3 \pm 0.6$. The rms of the RV fit is $56 \mathrm{~m} \mathrm{~s}^{-1}$ for a semiamplitude of $K_{1}=2.01 \pm$ $0.01 \mathrm{~km} \mathrm{~s}^{-1}$, which is comparable to the original fit (rms = $51 \mathrm{~m} \mathrm{~s}^{-1}$ for $K_{1}=2.08 \pm 0.04 \mathrm{~km} \mathrm{~s}^{-1}$ ).

\subsection{Dynamical Masses}

From the data summarized in Table 3, one can determine the dynamical masses of both components of the system. The revised
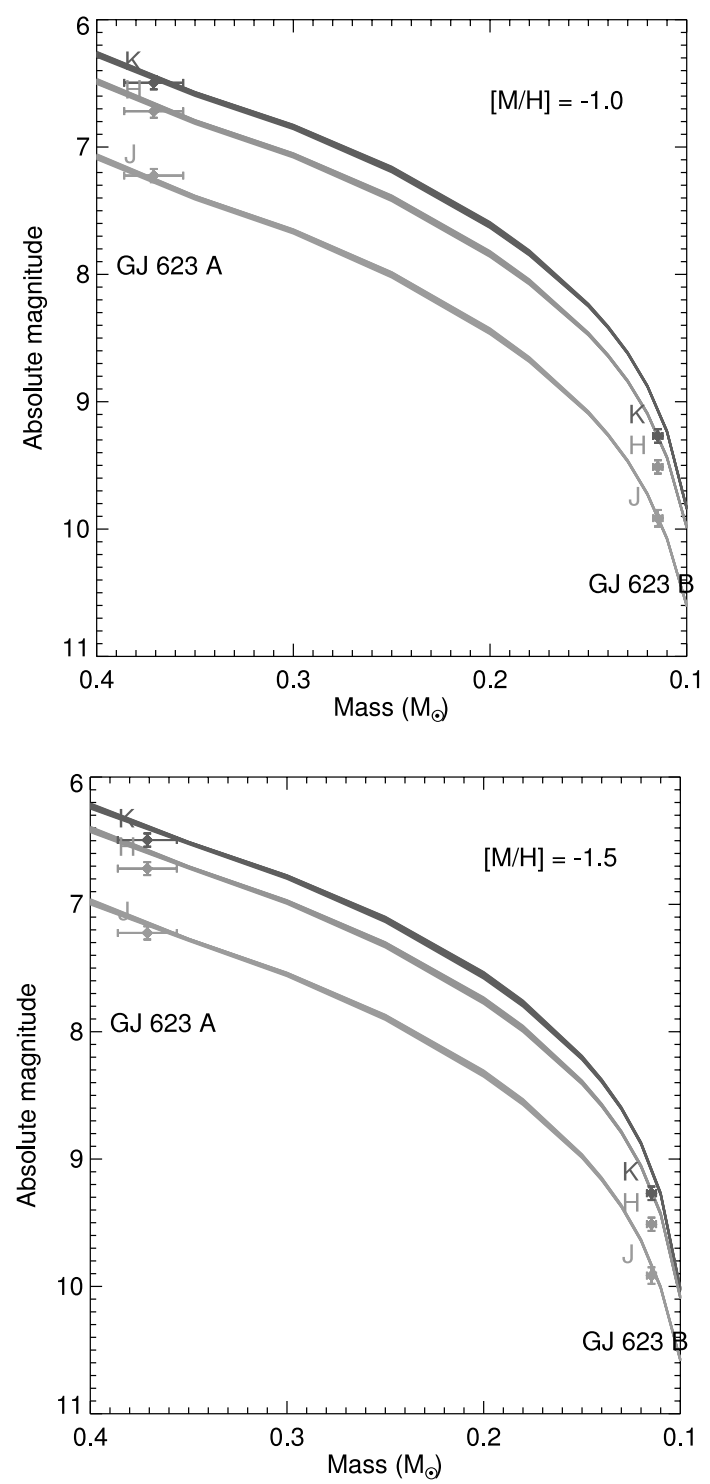

parallax figuring in the table takes the binarity into account. The semimajor axis $a$, expressed in $\mathrm{AU}$, once combined with the orbital period, gives the total mass $M_{T}$ and the associated uncertainty $\sigma_{T}$ :

$$
\begin{aligned}
M_{T} & =a^{3} / P^{2}, \\
\sigma_{T} / M_{T} & =\sqrt{\left(3 \sigma_{a} / a\right)^{2}+\left(2 \sigma_{P} / P\right)^{2}} .
\end{aligned}
$$

The likelihood analysis also determines the "pseudoamplitude" defined by equation (17) and its associated uncertainty.

This composite parameter, combined with the total mass, allows independent determination of the mass of the secondary $M_{2}$ :

$$
\begin{aligned}
M_{2} & =M_{T} \frac{A_{1} P}{2 \pi a}=\frac{A_{1} a^{2}}{2 \pi P}, \\
\sigma_{2} / M_{2} & =\sqrt{\left(\sigma_{A_{1}} / A_{1}\right)^{2}+\left(\sigma_{P} / P\right)^{2}+\left(2 \sigma_{a} / a\right)^{2}},
\end{aligned}
$$
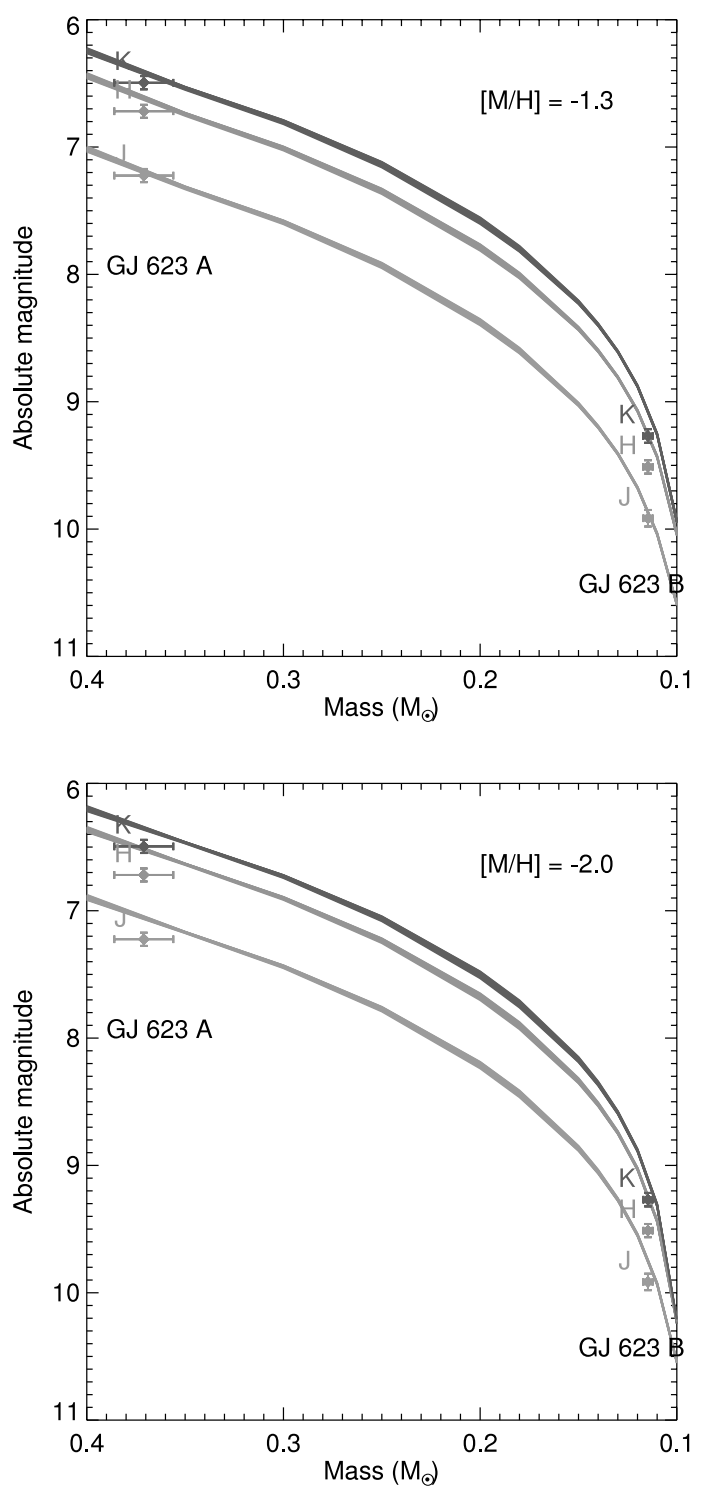

Fig. 5.-Mass-luminosity diagram for the GJ 623 system in $J, H$, and $K$ bands. Comparison with the low-mass population II star models by Montalbán et al. (2000) in the $0.1-0.4 M_{\odot}$ range, for different metallicities. [See the electronic edition of the Journal for a color version of this figure.] 
and the mass ratio $M_{2} / M_{T}$ :

$$
\begin{aligned}
M_{R} & =\frac{m_{2}}{M_{T}}=\frac{A_{1} P}{2 \pi a}, \\
\sigma_{R} / M_{R} & =\sqrt{\left(\sigma_{A_{1}} / A_{1}\right)^{2}+\left(\sigma_{P} / P\right)^{2}+\left(\sigma_{a} / a\right)^{2}} .
\end{aligned}
$$

A similar analysis is possible for $M_{1}$. Errors on the masses are dominated by the uncertainty on the semimajor axis, itself dominated by the error on the Hipparcos parallax. As a consequence, with a fractional error of $1 \%$, the mass ratio is better constrained than the mass of the secondary. The dynamical masses of the GJ 623 system are summarized in Table 4.

\subsection{Color, Metallicity, and Kinematics}

The multiwavelength contrast ratios found in Table 2 may be used to decompose the observed combined magnitude of the binary system into magnitudes for individual components. One determines the following absolute magnitudes: $M_{J}=7.224 \pm$ $0.052, M_{H}=6.719 \pm 0.051$, and $M_{K}=6.495 \pm 0.052$ for GJ $623 \mathrm{~A}$ and $M_{J}=9.915 \pm 0.065, M_{H}=9.512 \pm 0.052$, and $M_{K}=$ $9.269 \pm 0.053$ for GJ 623B.

With $J-K$ color indices of $0.729 \pm 0.074$ and $0.646 \pm$ 0.084 , respectively, for the primary and the secondary, the GJ $623 \mathrm{AB}$ system is bluer than the Delfosse et al. (2000) empirical $M-L$ relation. Therefore, we suspect GJ 623 is of low metallicity. From the Hipparcos proper motion $(1145.38,-452.37)$ mas yr $^{-1}$ (Perryman et al. 1997) and the RV offset $V_{0}=-27.4 \mathrm{~km} \mathrm{~s}^{-1}$ determined from our model fit, one can calculate the Galactic space velocity $(U, V, W)=(-33,14,-41) \mathrm{km} \mathrm{s}^{-1}$ after correction for standard solar motion. ${ }^{5}$ This velocity is consistent with an old disk population, and therefore of subsolar metallicity (Chiba \& Beers 2000), which is consistent with both components being bluer than the average field object.

5 The sign convention is the one of the IDL astrolib gal_uvw procedures. Note that the literature is confusing on this matter. For instance, McCarthy \& Henry (1987) provide numbers of comparable magnitude but with opposite sign for all $(U, V, W)$ components.
Figure 5 compares the location of both components of the GJ 623 system in a mass-luminosity diagram to the low-mass population II models of Montalbán et al. (2000) for different metallicities. The trend we observe with these models (cf. Fig. 5) supports GJ $623 \mathrm{AB}$ being of subsolar metallicity. The model that best matches our measurements for the primary is for $[\mathrm{M} / \mathrm{H}]=$ -1.0 . For the secondary, the best model predicts a slightly lower mass of $0.110 \pm 0.001 M_{\odot}$ for $[\mathrm{M} / \mathrm{H}]=-2.0$. This large discrepancy in metallicity is inconsistent with the assumption of a coeval binary. The very low metallicity for the secondary would be consistent with GJ 623B belonging to the Galactic halo, which is unlikely according to the kinematics. Therefore, we conclude that the models do not adequately fit the data.

\section{CONCLUSION}

The observation of binary systems is the only way to measure unbiased masses. As shown in this paper, combined with excellent radial velocity measurements, a few high-angular resolution images provide sufficient information to constrain the range of possible masses below the $5 \%$ precision that is required to seriously challenge the models at the low end of the main sequence.

The application of precision RV methods developed for planet searches combined with AO will provide a complete characterization of the stellar structure of the lower main sequence.

We thank the staff and telescope operators of Palomar Observatory and Keck Observatory for their support. F. M. thanks Terry Herter for his help. This work is partially funded by the National Science Foundation under grants AST 03-35695 and AST 05-06588. This publication makes use of the Simbad database, operated at CDS, Strasbourg, France and the data products from the Two Micron All Sky Survey, which is a joint project of the University of Massachusetts and the Infrared Processing and Analysis Center/California Institute of Technology, funded by the National Aeronautics and Space Administration and the National Science Foundation. We wish to extend special thanks to those of Hawaiian ancestry on whose sacred mountain we are privileged to be guests. Without their generous hospitality, the observations presented herein would not have been possible.
Baldwin, J. E., Haniff, C. A., Mackay, C. D., \& Warner, P. J. 1986, Nature, 320, 595

Baraffe, I., Chabrier, G., Allard, F., \& Hauschildt, P. H. 1998, A\&A, 337, 403

Barbieri, C., Demarchi, G., Nota, A., Corrain, G., Hack, W., Ragazzoni, R., \& Macchetto, D. 1996, A\&A, 315, 418

Chiba, M., \& Beers, T. C. 2000, AJ, 119, 2843

Delfosse, X., Forveille, T., Ségransan, D., Beuzit, J.-L., Udry, S., Perrier, C., \& Mayor, M. 2000, A\&A, 364, 217

Delfosse, X., et al. 2004, in ASP Conf. Ser. 318, Spectroscopically and Spatially Resolving the Components of the Close Binary Stars, ed. R. W. Hilditch, H. Hensberge, \& K. Pavlovski (San Francisco: ASP), 166

Eisner, J. A., \& Kulkarni, S. R. 2002, ApJ, 574, 426

Hayward, T. L., Brandl, B., Pirger, B., Blacken, C., Gull, G. E., Schoenwald, J., \& Houck, J. R. 2001, PASP, 113, 105

Henry, T. J. 1998, in ASP Conf. Ser. 134, Brown Dwarfs and Extrasolar Planets, ed. R. Rebolo, E. L. Martin, \& M. R. Zapatero Osorio (San Francisco: ASP), 28

Henry, T. J., Franz, O. G., Wasserman, L. H., Benedict, G. F., Shelus, P. J., Ianna, P. A., Kirkpatrick, J. D., \& McCarthy, D. W., Jr. 1999, ApJ, 512, 864 Jancart, S., Jorissen, A., Babusiaux, C., \& Pourbaix, D. 2005, A\&A, 442, 365 Lippincott, L. S., \& Borgman, E. R. 1978, PASP, 90, 226

Lloyd, J. P., Martinache, F., Ireland, M. J., Monnier, J. D., Pravdo, S. H., Shaklan, S. B., \& Tuthill, P. G. 2006, ApJ, 650, 131
Marcy, G. W., \& Moore, D. 1989, ApJ, 341, 961

McCarthy, D. W., Jr., \& Henry, T. J. 1987, ApJ, 319, L93

Metchev, S. A., \& Hillenbrand, L. A. 2004, ApJ, 617, 1330

Montalbán, J., D’Antona, F., \& Mazzitelli, I. 2000, A\&A, 360, 935

Nakajima, T., Kulkarni, S. R., Gorham, P. W., Ghez, A. M., Neugebauer, G., Oke, J. B., Prince, T. A., \& Readhead, A. C. S. 1989, AJ, 97, 1510

Nidever, D. L., Marcy, G. W., Butler, R. P., Fischer, D. A., \& Vogt, S. S. 2002, ApJS, 141, 503

Perryman, M. A. C., et al. 1997, A\&A, 323, L49

Pourbaix, D., et al. 2004, A\&A, 424, 727

Pravdo, S. H., Shaklan, S. B., Wiktorowicz, S. J., Kulkarni, S., Lloyd, J. P., Martinache, F., Tuthill, P. G., \& Ireland, M. J. 2006, ApJ, 649, 389

Racine, R., Walker, G. A. H., Nadeau, D., Doyon, R., \& Marois, C. 1999, PASP, 111, 587

Readhead, A. C. S., Nakajima, T. S., Pearson, T. J., Neugebauer, G., Oke, J. B., \& Sargent, W. L. W. 1988, AJ, 95, 1278

Ségransan, D., Delfosse, X., Forveille, T., Beuzit, J.-L., Udry, S., Perrier, C., \& Mayor, M. 2000, A\&A, 364, 665

Tuthill, P. G., Monnier, J. D., Danchi, W. C., Wishnow, E. H., \& Haniff, C. A. 2000, PASP, 112, 555

van Leeuwen, F., \& Evans, D. W. 1998, A\&AS, 130, 157 